\title{
ORDINAL CLASSIFICATION FOR EFFICIENT PLANT STRESS PREDICTION IN HYPERSPECTRAL DATA
}

\author{
J. Behmann*, P. Schmitter, J. Steinrücken, L. Plümer
}

Institute of Geodesy and Geoinformation, University of Bonn, Meckenheimer Allee 172, Bonn, Germany, behmann@igg.uni-bonn.de

\author{
Commission VII, WG VII/3
}

KEY WORDS: Hyper spectral, Classification, Close Range, Agriculture, Feature

\begin{abstract}
:
Detection of crop stress from hyperspectral images is of high importance for breeding and precision crop protection. However, the continuous monitoring of stress in phenotyping facilities by hyperspectral imagers produces huge amounts of uninterpreted data. In order to derive a stress description from the images, interpreting algorithms with high prediction performance are required. Based on a static model, the local stress state of each pixel has to be predicted. Due to the low computational complexity, linear models are preferable.

In this paper, we focus on drought-induced stress which is represented by discrete stages of ordinal order. We present and compare five methods which are able to derive stress levels from hyperspectral images: One-vs.-one Support Vector Machine (SVM), one-vs.-all SVM, Support Vector Regression (SVR), Support Vector Ordinal Regression (SVORIM) and Linear Ordinal SVM classification. The methods are applied on two data sets - a real world set of drought stress in single barley plants and a simulated data set. It is shown, that Linear Ordinal SVM is a powerful tool for applications which require high prediction performance under limited resources. It is significantly more efficient than the one-vs.-one SVM and even more efficient than the less accurate one-vs.-all SVM. Compared to the very compact SVORIM model, it represents the senescence process much more accurate.
\end{abstract}

\section{INTRODUCTION}

Crop stress is induced by environmental factors (e.g. drought, out-of-range temperatures or pathogens) which exceed a critical level (Gaspar et al., 2002, Taiz and Zeiger, 2010). Under prolonged stress, crop productivity is impaired significantly (Gaspar et al., 2002). In order to meet the demand of agricultural output for an increasing world population (FAO, 2009), agricultural science is challenged to enhance crop productivity by improving methods of crop management (Davies et al., 2011) and by breeding crops with higher stress tolerance levels (Tester and Langridge, 2010). Breeding and crop management will benefit from phenotyping information: the detection, quantification and visualization of a plant's stress responses.

In this paper, we focus on drought stress, one of the biggest challenges in global crop production (Pennisi, 2008, Tuberosa and Salvi, 2006). If water shortage exceeds a critical level, a plant initiates stress responses which result in biochemical and morphological adaptations. An important response process, in which resources are reallocated within the plant, is leaf senescence. Leaf senescence denotes the final phase of leaf development and may be induced prematurely under drought stress (Lim and Nam, 2007). It is a spatiotemporal process, which allows the plant to attain the reproductive state under drought conditions. The process is characterized by a degradation of pigments and the relocation of nutrients. It develops continuously and proceeds in patterns from older to younger leaves and, within a leaf, from the tip towards the leaf base (Guiboileau et al., 2010, Lim and Nam, 2007). Furthermore, the senescence process forms an ordinal order mainly related to pigment degradations (Merzlyak et al., 1999).

In contrast to some plant diseases, drought stress induced senescence does not manifest itself in local symptoms. The reallocation of resources involves the entire plant - and occurs in all

\footnotetext{
${ }^{*}$ Corresponding author
}
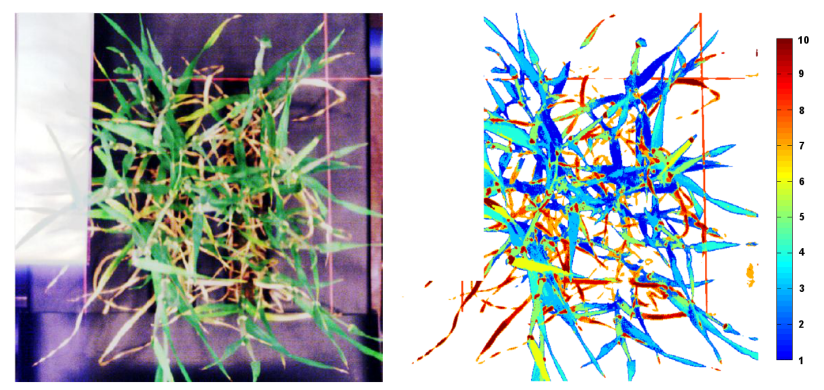

Figure 1: RGB visualization and labeling of a hyperspectral image of an barley plant.

plants, even the well watered, to a specific degree. Drought stressed plants are characterized by early and accelerated leaf senescence (Munné-Bosch and Alegre, 2004). The aforementioned degradation of pigments (particularly chlorophyll) alters the ratio between reflected, absorbed and transmitted radiation (Blackburn, 2007). These changes in spectral characteristics can be observed non-invasively by hyperspectral sensors - even in early stages. The detection and distinction from normal variations requires spectral information with high degrees of temporal and spatial resolution.

The analysis of such series of hyperspectral images is challenging - especially in real-time applications. The occurrence of different degrees of leaf senescence in a single plant requires analysis methods which predict the stress state for each pixel. The aggregation of these local states compose a global pattern which allows conclusions about a plant's health state (Fig. 1). On pixel scale the early stress stages are invisible for the human eye and, therefore, labels are extracted by an unsupervised labeling (Behmann et al., 2014). The continuous senescence process is discretized into classes which are ordered on an ordinal scale. The contextual knowledge about this ordinal scale can be integrated into the 
model selection resulting in adapted and more efficient prediction methods.

In this paper, we present an evaluation of five supervised prediction methods for deriving the local stress levels: One-vs.-one Support Vector Machine (SVM), one-vs.-all SVM, Support Vector Regression (SVR), Support Vector Ordinal Regression (SVORIM) and Linear Ordinal SVM classification. In order to compare their accuracy and efficiency, the methods are applied on two data sets - a real world set of drought stress in barley (Hordeum vulgare) and a simulated data set. In the barley data set, the spectra are represented by the values of five Vegetation Indices (VIs).

The rest of this paper is organized as follows: In Section 2 we will describe the data sets used; the aforementioned prediction algorithms will be introduced in Section 3. In the fourth section, the results of applying the algorithms on the data sets are presented and discussed. The paper ends in Section 5 with a conclusion.

\section{DATA SET}

In this study, we compare the performance of different prediction algorithms on two data sets. The first data set consists of simulated features and partial overlapping classes with a perfect ordinal order. The second data set consists of VIs derived from hyperspectral images of barley plants under drought stress. The selection of these data sets intends to show the theoretical advantages of ordinal classification and how much benefit remains in real world applications.

\subsection{Simulated ordinal data}

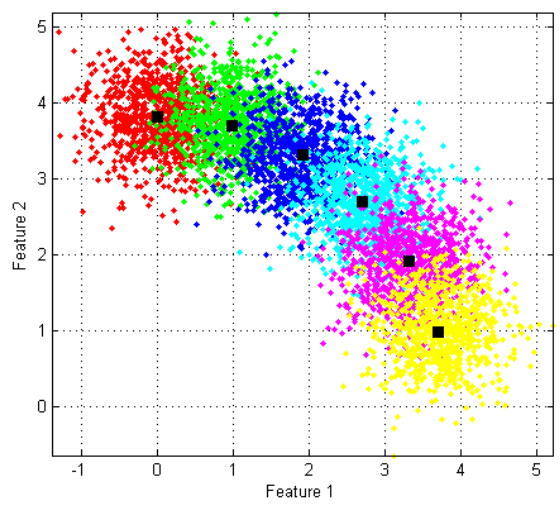

Figure 2: The simulated ordinal data set consists of six Gaussian distributed classes. Each class is represented by a different color, the centroids of the classes are represented by black squares.

The simulated data set consists of six classes and represents a prototype of ordinal ordered data. It is used to visualize the discriminant functions of the applied prediction algorithms and to show their relevant differences. In order to enable the visualization of the whole feature space, it contains only two features. The ordinal structure is realized by arranging the classes on an arc as shown in Fig. 2. The class centers $c_{i}$ have the same pair-wise distance of 1 and the samples are Gaussian distributed by $\sim N\left(c_{i}, 0.2\right)$. The standard deviation of 0.2 maintains the ordinal order of the classes and, on the other hand, allows distinguishing different result qualities. The data set consists of 5000 labeled instances; $10 \%$ are used as training data, the remaining as test data.

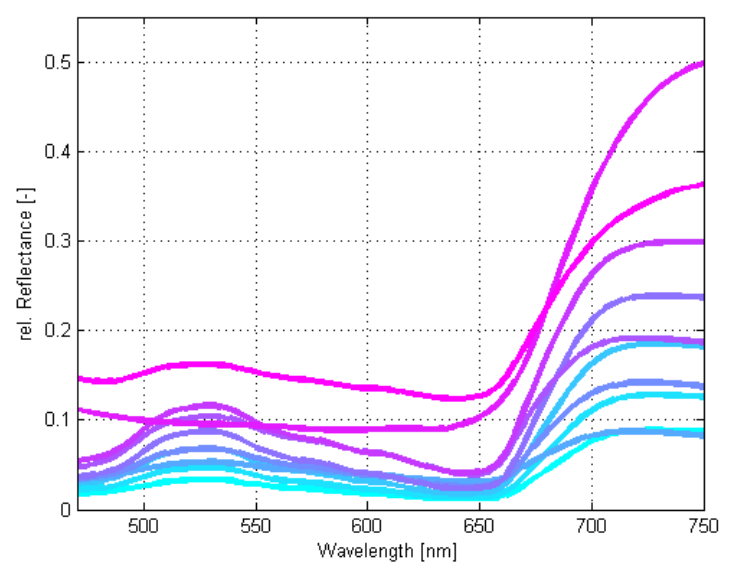

Figure 3: Centroids of the cluster for the labeling of the barley data set. The transition from blue to magenta represents the senescence states of the corresponding spectra.

\subsection{Hyperspectral features of drought stressed barley plants}

The real-world data set is derived from time series of hyperspectral images which have been described in detail in (Behmann et al., 2014). In that study, we aimed to detect drought stress induced changes in single barley plants as early as possible. Hyperspectral images were recorded daily for a period of 20 days by a SOC700 hyperspectral imager (Surface Optics, USA). The SOC700 observes the reflectance characteristics from $430 \mathrm{~nm}$ to $890 \mathrm{~nm}$ in 120 bands; each hyperspectral image has a spatial resolution of $640 \times 640$ pixels. The images were preprocessed by removing the background using a combination of clustering and setting a threshold as described in (Behmann et al., 2014). Furthermore, the spectral range is reduced due to noise effects at spectral border regions. Examples of hyperspectral images and the spatial variability of the senescence process are shown in Fig. 1 and Fig. 9.

The pixels are labeled by an unsupervised labeling, introduced in (Behmann et al., 2014). The unsupervised labeling uses k-Means to extract $k$ ordinal ordered classes whose centroids represent the ordinal order mainly related to chlorophyll degradations of the senescence process. The classes are labeled in ascending order from 1 to $k$ and the labels are assigned to single pixels.

In this study, the labeling uses $k=10$ classes and the instances were sampled without spatial context. The final barley data set comprises 211500 test and 21150 training instances, each represented by the values of five Vegetation Indices (VIs). The used VIs were selected by the ReliefF algorithm (Kononenko, 1994) from a basic feature set of 20 VIs (Exelis Visual Information Solutions, 2012) to reliably exclude irrelevant features and are given in Tab. 1.

\begin{tabular}{c|c|c} 
Name & Formula & Reference \\
\hline ARVI & $\frac{R_{800}-2\left(R_{670}-R_{490}\right)}{R_{800}+2\left(R_{670}-R_{490}\right)}$ & (Kaufman and Tanré, 1996) \\
RGRI & $\frac{\operatorname{Mean}\left(R_{500-600}\right)}{\operatorname{Mean}\left(R_{600}-700\right)}$ & (Gamon and Surfus, 1999) \\
RENDVI & $\frac{R_{750}-R_{705}}{R_{750} R_{705}}$ & (Gitelson and Merzlyak, 1994) \\
SumGreen & $\frac{1}{n} \sum_{i=500}^{599} R_{i}$ & (Gamon and Surfus, 1999) \\
PSRI & $\frac{R_{680}-R_{500}}{R_{750}}$ & (Merzlyak et al., 1999)
\end{tabular}

Table 1: The selected VIs included in the barley data set ordered descending by their ReliefF score 


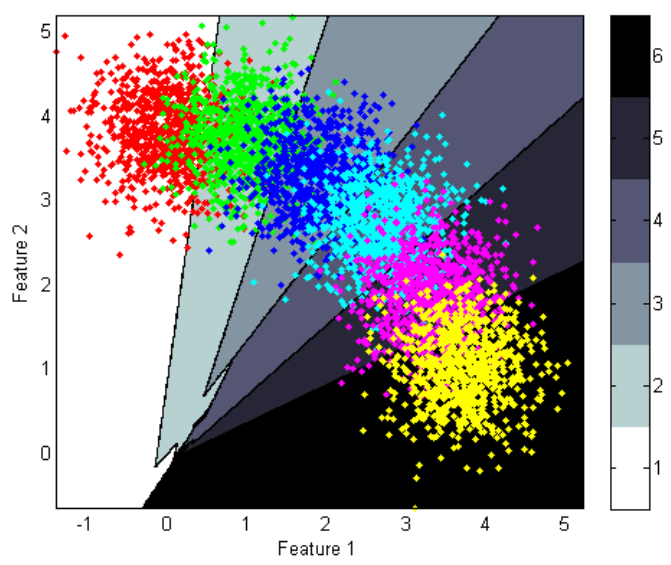

Figure 4: Decision boundaries of the one-vs.-one SVM classification model for the simulated data set

\section{PREDICTION ALGORITHMS}

In machine learning, various supervised algorithms are available for the deduction of models from annotated/labeled training data and the prediction of target variables for unlabeled test data. Classification algorithms predict discrete classes whereas regression algorithms predict continuous target values. Ordinal classification relies on the assumption of ordinal ordered but discrete classes with a corresponding structure in the feature space.

\subsection{Multiclass SVM classifiers}

The Support Vector Machine (SVM) (Cortes and Vapnik, 1995) is an established classification method that determines the optimal, linear discriminant function between two classes based on the maximum margin principle. Extensions of this method handle overlapping classes and even non-linear discriminant functions. Multi-class tasks are handled in general by decomposing the multi-class problem in multiple binary class problems (Duan and Keerthi, 2005). The most common decomposing approaches are the one-vs.-one and the one-vs.-all approach.

3.1.1 One-vs.-one SVM The one-vs.-one SVM is the most common multiclass approach. It is based on pairwise classification, separating all classes from each other (Fürnkranz, 2002). An example of the decision boundaries for the simulated data set is shown in Fig. 4.

In the learning step, a discrimination function is optimized for each class pair resulting in $\frac{n *(n-1)}{2}$ discrimination functions for $n$ classes. Each optimization uses only the training samples of the regarded pair of classes. The optimization is quite efficient because the amount of training data for a single optimization is small (Duan and Keerthi, 2005). However, the number of optimization procedures increases quadratically with the number of classes. A high number of classes result in many, potential unnecessary, discriminate function.

The classification follows the max wins voting principle in which each discrimination function is applied to the sample (Duan and Keerthi, 2005). Every winning class gets a vote and the class with the highest number of votes is selected as predicted class. This principle is very robust because the contribution of a single, probably misleading discriminant function, is limited. However, for each prediction all of the $\frac{n *(n-1)}{2}$ discrimination function have to be evaluated. For an improved prediction performance, approaches which can reduce the application of discrimination functions (e.g. directed acyclic graph SVM) were proposed (Platt et al., 1999).

3.1.2 One-vs.-all SVM The one-vs.-all approach consists of discrimination functions which separate one class from all other classes, wherefore it is also called one-vs.-the-rest approach. The discriminant functions are determined by separating the training samples of one class from the aggregated training samples of all other classes. An example of the decision boundaries for the simulated data set is shown in Fig. 5. The model is more compact as only $n$ discriminant functions are needed to separate $n$ classes (Duan and Keerthi, 2005). The classification is based on the winner takes all principle, where the instance is assigned to the class with the maximum probability (Platt, 1999) or, alternatively, the highest classification score (normalized distance to the discriminant function). Using posterior probabilities, a stochastic interpretation is enabled and in some applications accuracy improvements are possible. On the other hand, the determination of the sigmoid functions is computational expensive and additional parameters are required. Therefore, the use of the classification score is preferred in this study focusing the prediction performance.

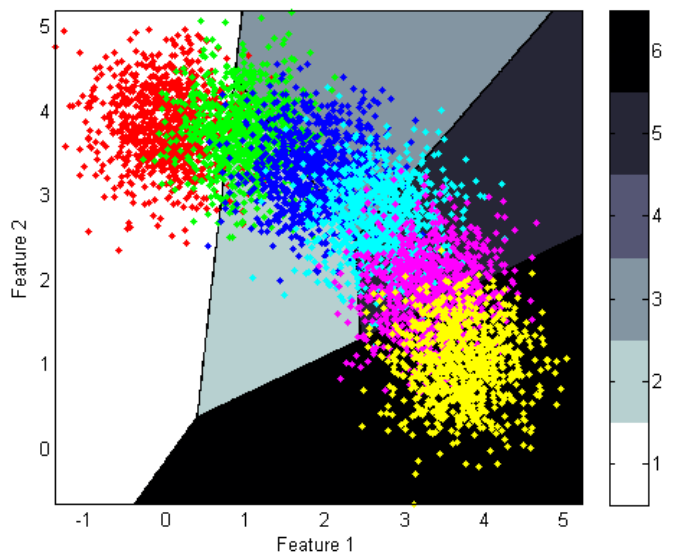

Figure 5: Decision boundaries of the one-vs.-one SVM classification model for the simulated data set

The one-vs.all multiclass approach is less common than the onevs.-one. It is less robust against outliers because a single misleading discriminant function can impair the result quality significantly (Duan and Keerthi, 2005). However, using well-tuned SVM classifiers comparable result qualities are achievable (Rifkin and Klautau, 2004). Each of the binary discriminant functions suffers from a class imbalance since one class is separated from all the others. Moreover, the usage of all training data for each optimization can reduce the performance in the training step (Duan and Keerthi, 2005). Whereas in the one-vs.-one approach only two classes contribute to a discriminant function, in the one-vs.all approach all classes contribute to all discriminant functions. However, the number of SVM evaluations is lower than in the one-vs.-one approach: each of the discriminant functions has to be evaluated for a prediction but the number of functions is lower, especially for higher numbers of classes.

\subsection{Support Vector Regression}

The main difference between Support Vector Regression (SVR) and SVM is the type of target variable. Regression algorithms predict continuous, real-valued labels in contrast to the discrete classes of classification models (Smola and Schölkopf, 2004). This is reflected in the optimization algorithm which adapts the 


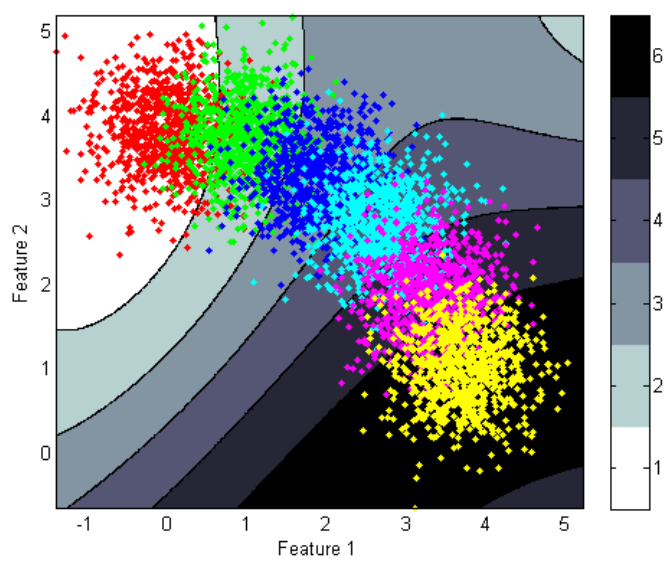

Figure 6: Decision boundaries of the Support Vector Regression model at the simulated data set. The continuous predictions are rounded to an integer to extract the decision boundaries.

basic principle of the binary SVM and results in similar formulas (Vapnik et al., 1997). The formulation is generalizable to nonlinear applications by the well-known kernel trick which implicitly maps the feature vectors $x_{j}$ to a higher dimensional feature space and determines their distance $K\left(x_{i}, x_{j}\right)$ in this space.

The regression function is parameterized by the support vectors (SVs) $x_{i}$, the Lagrangian coefficients $\alpha_{i}^{*}$ and $\alpha_{i}$ and the offset $b$ to

$$
y=f\left(x, \alpha, \alpha^{*}\right)=\sum_{i=1}^{l}\left(\alpha_{i}^{*}-\alpha_{i}\right) K\left(x, x_{i}\right)+b .
$$

The primal optimization function shows the regression approach of the SVR. It is searched for a function that deviates up to a distance $\epsilon$ for most of the trainings samples and is as flat as possible (Smola and Schölkopf, 2004). The flatness maximizes the robustness against variations of single features of the input vector $x_{i}$. The complexity of the regression model is controlled by the parameters: tolerance $\epsilon$, error weight $C$ and potentially additional kernel parameters.

The SVR was designed to find a regression function based on training instances which are continuously distributed in the feature values as well as the labels (Smola and Schölkopf, 2004). The discrete classes of the ordinal data sets aggregate a high number of instances to a single label value and request a step function. The SVR may approximate the function but its smoothness condition will smooth out the step borders. The SVR is able to model also ordinal data sets but the approximation errors will reduce the prediction quality for ordinal classification data sets. On the other hand, the smooth transition between the classes can be used to represent the uncertainty at the class borders without explicit probability modeling.

The kernels provide linear and non-linear model types. The linear SVR is an extremely compact model but achieves an inferior accuracy for both data sets. Therefore, we applied the SVR with a radial basis function (rbf) kernel. This model is able to represent the ordinal transition with a competitive accuracy (Fig. 6). The increased accuracy is accompanied by a higher model complexity due to the non-linear kernel function.

\subsection{Ordinal classification}

The ordinal classification is applicable in scenarios with discrete labels and known class order (Dembczyński et al., 2008). As it is a special case of the general multi-class scenarios, the introduced general prediction methods can be used. Prediction methods which are more adapted to the ordinal structure utilize the additional knowledge about the data set (Behmann et al., 2014) and achieve higher performance measurements. In general, the information about the ordinal data structure is used to reduce the model size by omitting model parts which are not required (Chu and Keerthi, 2007). Different approaches were developed which differ in specific assumptions on data characteristics and the robustness against non-ordinal aspects.

3.3.1 Support Vector Ordinal Regression Support Vector Ordinal Regression (SVORIM) was developed by (Chu and Keerthi, 2007) in an explicit and an implicit formulation. Both formulations determine $c-1$ parallel hyperplanes that separate $c$ classes and preserves the natural ordinal ordering. The parallelism of the hyperplanes reduces model size and complexity significantly. The linear model comprises only a single weight vector $w$ for the whole model and a threshold $b_{i}$ for each of the $c-1$ hyperplanes. The optimization is conducted by an adapted sequential minimal optimization (SMO) algorithm, optimizing the ranking of the training instances (Chu and Keerthi, 2007).

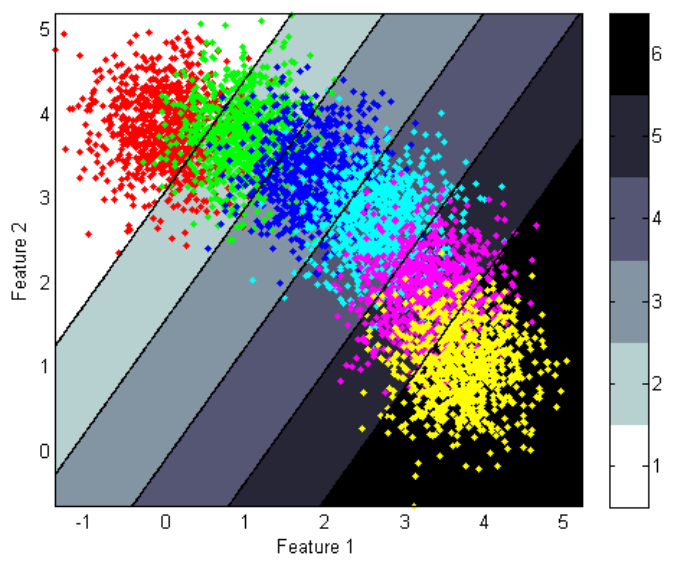

Figure 7: Support Vector Ordinal Regression is characterized by parallel discriminant functions resulting in a very compact model.

Fig. 7 shows the position and the orientation of the discriminant functions for the simulated data set. In this context, it becomes apparent that the model is limited regarding non-linear ordinal processes or non-ordinal aspects. However, the SVORIM prediction step is extremely efficient and comprises only a multiplication with the weight vector and the application of the $c-1$ thresholds. The Support Vector Ordinal Regression represents the most compact model with the lowest model complexity but the low complexity is accompanied by a low adaptability to deviating data characteristics. This may reduce the prediction accuracy on real-world data sets.

3.3.2 Linear Ordinal SVM classification The Linear Ordinal classification is defined by discriminant functions between classes which are neighboring on the ordinal scale like at the Support Vector Ordinal Regression (Chu and Keerthi, 2007). Deviating from this approach, the hyperplanes are not forced to be parallel but are optimized locally (Dembczyński et al., 2008). The number of discriminant function remains at $c-1$ but the number of model parameter is significantly higher because each discriminant function has an individual weight vector $w_{i}$ (Behmann et al., 2014).

Figure 8 shows the discriminant functions for the simulated data set. The improved flexibility of the model is apparent but in the 


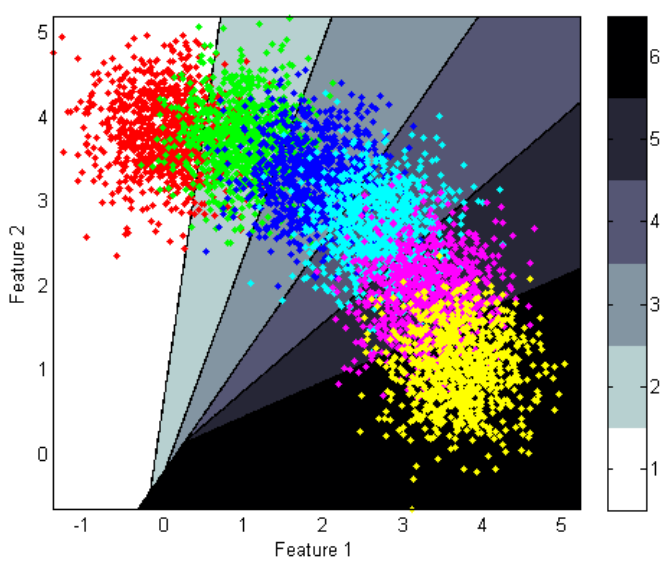

Figure 8: Decision boundaries of the Linear Ordinal SVM classification model for the simulated data set. The overlapping discriminant functions are combined by a decision tree for an unambiguous result.

regions without training instances, the discriminant functions intersect each other. Without additional information, these regions of intersections are undefined. Therefore, a tree structure is introduced to unambiguously assign a class for each part of the feature space (Behmann et al., 2014). In the tree structure a hierarchy of classes is established by an interval bisection approach. The discriminant functions can be represented by various classification approaches, e.g. SVM, random forests, logistic regression or naive Bayes.

In this study, we used linear SVMs to enable a reliable comparability to the other approaches. In the training step, each discriminant function is optimized on its own with its individual SVM parameter

$C_{i}$

(Behmann et al., 2014). The Linear Ordinal SVM classification is represented by the aggregate of all discriminant functions and the tree structure is used for class prediction. In the prediction, the number of evaluation steps is reduced by using the tree structure. Starting from the tree root, the classification is done in $\log (c)$ steps.

The concept of Linear Ordinal SVM lies between the flexible one-vs.-one multi-class approach and the extreme compact but inflexible Support Vector Ordinal Regression (Chu and Keerthi, 2007). It is able to represent also non-linear ordinal processes but it still relies on the ordinal data characteristics. Non-ordinal aspects cannot be represented due to the reduced number of discriminant functions compared to the generic multi-class approaches. The Linear Ordinal SVM classification results in much more compact models compared to one-vs.-one classification and may adapt to real-world data sets with only slight losses in accuracy.

\section{RESULTS AND DISCUSSION}

We evaluate the presented prediction algorithms on two different data sets. The simulated data set contains ordinal classes in a two-dimensional visualizable feature space. The barley data set contains pixel values with five VIs as features and a ordinal senescence class. This real world data may contain minor nonordinal aspects and the prediction algorithms have to deal with significant noise effects.

\subsection{Simulated ordinal data}

For the simulated data set, the results of the prediction algorithms are very close with the exception of the one-vs.-all SVM (Fig.
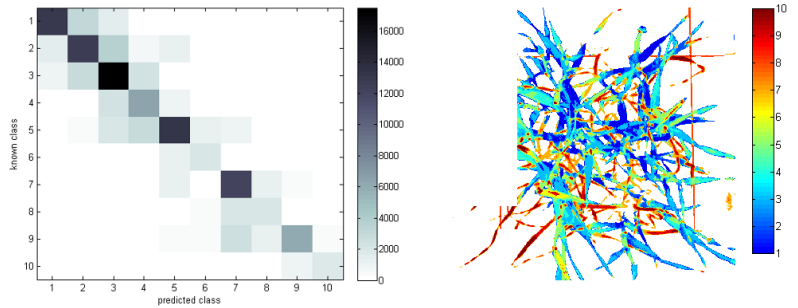

Figure 9: Confusion matrix and predicted labels by the Linear Ordinal SVM for a hyperspectral image of a barley plants

10 and Tab. 2). The visualization in Fig. 5 shows that the underlying linear model is not able to separate a single class from the remaining classes. As a result, only the classes 1 and 6 are classified correctly, whereas the remaining classes are classified at random. This effect appears always, if a class is not linearly separable from the remaining classes which is in many cases related to a disproportion between the number of features and the number of classes (2 against 6 in the simulated data set). Slight drawbacks are visible at the SVORIM classification which is not flexible enough to follow the arch-shaped ordinal class distribution. The limitation to parallel decision functions requires data with a linear development in each of the features over the whole ordinal structure. The SVR achieves good results for the simulated data set due to the flexible rbf kernel. However, the model size increases drastically which impedes the competitiveness to the other prediction methods regarding prediction efficiency (Tab. 2). The one-vs.-one SVM and the Linear Ordinal SVM are simi-
(1)

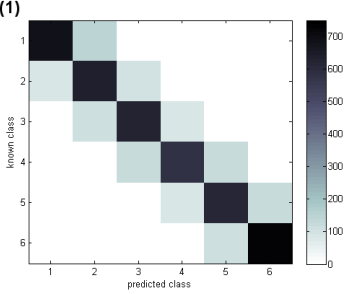

(3)
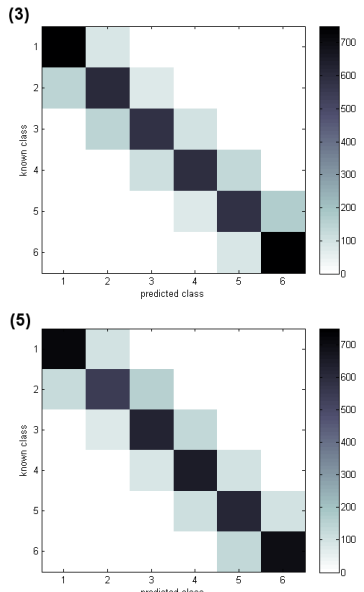

(2)

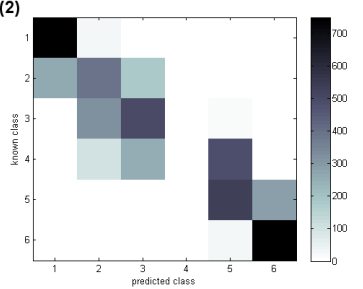

(4)

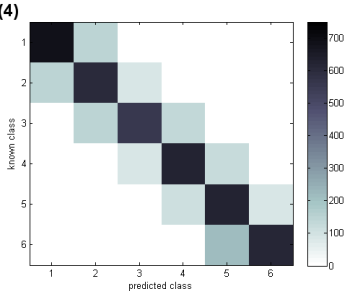

Confusion matrices

(1) one-vs. -one SVM

(2) one-vs.-all svm

(3) Support Vector Regression

(4) Support Vector Ordinal Regression

(5) Linear ordinal SVM
Figure 10: Confusion matrix of the prediction algorithms for the simulated data set with a pure ordinal structure.

lar regarding accuracy and also the positions of class boundaries (Fig. 4 and 8 ). This is caused by the characteristic of both methods to use pairwise discriminant functions. In case of the Linear Ordinal SVM the unneeded discriminant functions are omitted whereas the one-vs.-one approach derives discriminant functions between all pairs of classes. Different characteristics appear in the overlapping parts of the feature space. Here, the one-vs.-one approach decides based on class voting whereas the Linear Ordi- 


\begin{tabular}{l|cccc}
\hline Method & Accuracy [\%] & MSE & \# evaluation functions & \# model parameter \\
\hline one-vs.-one SVM & 78 & 0.22 & 15 & 45 \\
one-vs.-all SVM & 54 & 0.49 & 6 & 18 \\
Support Vector Regression (rbf) & 76 & 0.18 & 1 & 793 \\
Support Vector Ordinal Regression & 74 & 0.26 & 5 & 7 \\
Linear Ordinal SVM & 78 & 0.23 & 5 & 15 \\
\hline
\end{tabular}

Table 2: Performance overview on the simulated data set

\begin{tabular}{l|cccc}
\hline Method & Accuracy [\%] & MSE & \# evaluation functions & \# model parameter \\
\hline one-vs.-one SVM & 83 & 0.80 & 45 & 270 \\
one-vs.-all SVM & 46 & 1.90 & 10 & 60 \\
Support Vector Regression (rbf) & 66 & 0.72 & 1 & 43986 \\
Support Vector Ordinal Regression & 47 & 1.02 & 9 & 14 \\
Linear Ordinal SVM & 70 & 0.82 & 9 & 54 \\
\hline
\end{tabular}

Table 3: Performance overview on the barley data set

nal SVM uses a predefined tree structure.

The simulated data set is suitable to compare the different prediction algorithms and to highlight specific characteristics. As it shows a pure ordinal process the algorithms are compared under perfect conditions. In contrast, real world data sets contain noise, irrelevant processes and non-ordinal aspects. The performance of the algorithms depends significantly on the robustness against such deviations from perfect conditions.

\subsection{Data set: Senescence in barley}

The results for the barley data set represents the performance in real world applications (Fig. 11). The differences between the prediction algorithms increase compared to the simulated data set, presumably due to non-ordinal aspects (Tab. 3).
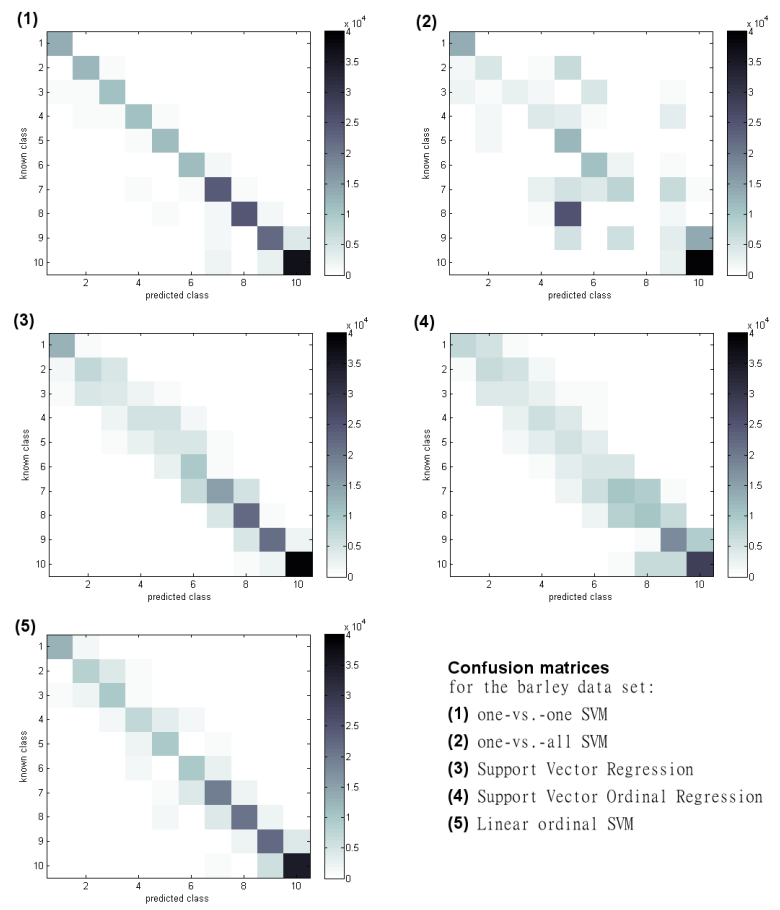

Figure 11: Confusion matrices for the described barley data set.

The prediction algorithms can be separated in two groups: a good accuracy is achieved by the on-vs.-one SVM (83\%), the SVR $(66 \%)$ and the Linear Ordinal SVM (70\%); an inferior accuracy is achieved by the on-vs.-all SVM (46\%) and the Support Vector Ordinal Regression (47\%).
The loss of accuracy of the SVORIM compared to the remaining methods is significant and is related to the data characteristics. The real-world data incorporating non-linear development of features over the ordinal scale cannot be described by parallel five-dimensional hyperplanes.

Again, the lowest accuracy is achieved by the one-vs.-all SVM. This effect is most probably related to the low number of features (five features and ten classes). Linear discriminant functions seem not to be able to separate one of the ten senescence classes from the others. This effect can be faced by using more features but this would increase data volume as well as model complexity.

The rbf SVR reaches competitive accuracy comparable to the one-vs.-one SVM and the Linear Ordinal SVM. The MSE value is the lowest of all methods related to the continuous predictions. Such output enables further evaluations like probability extraction and a more detailed visualization. However, its non-linear kernel increases the model size up to an factor of 800 . Such a model size prevents a high-throughput prediction as it is required for the efficient evaluation of hyperspectral images. Therefore, it is not suited to be applied for the introduced phenotyping scenario.

The one-vs.-one SVM and the Linear Ordinal SVM reach an almost identical MSE value. However, the accuracy of the one-vs.one approach is $13 \%$ higher. The combination of both result quality measurements reveals the classification characteristics. The one-vs.-one classifies more test samples correctly but if a test sample is misclassified it is more likely assigned to a more distant class. In contrast, the Linear Ordinal SVM assigns the misclassified samples in the most cases to one of the two neighboring classes. For the detection of disperse drought stress effects, the overall impression is most important (Fig. 9). It is not or only little affected by misclassifications to neighboring classes because these classes have nearly the same meaning with regard to the senescence level. Therefore, the higher accuracy of the one-vs.one SVM approach has only slight positive effects on real-world applications but this advantage is at the expense of a five times higher number of model parameters.

\subsection{Prediction efficiency for high-throughput phenotyping}

This study focuses the prediction efficiency needed for high throughput phenotyping systems. Such systems measure continuously the reflection characteristics of plants generating huge amounts of data. They require methods to compress quickly the observed data to valuable information. Fig. 12 opposes the reached accuracy to the number of model parameters related to the required prediction effort. The one-vs.-one method reaches the highest accuracy but needs 5 times more model parameters for $13 \%$ more 
accuracy compared to the Linear Ordinal SVM. Therefore, the user has to choose which characteristic is in the focus. The SVORIM approach is extremely fast but has significant drawbacks in accuracy whereas the one-vs.one SVM approach reaches the best accuracy using 20 times more model parameter. The Linear Ordinal SVM is a compromise between these extrema using a low number of model parameters and reaching an accuracy suitable for many applications (Behmann et al., 2014).

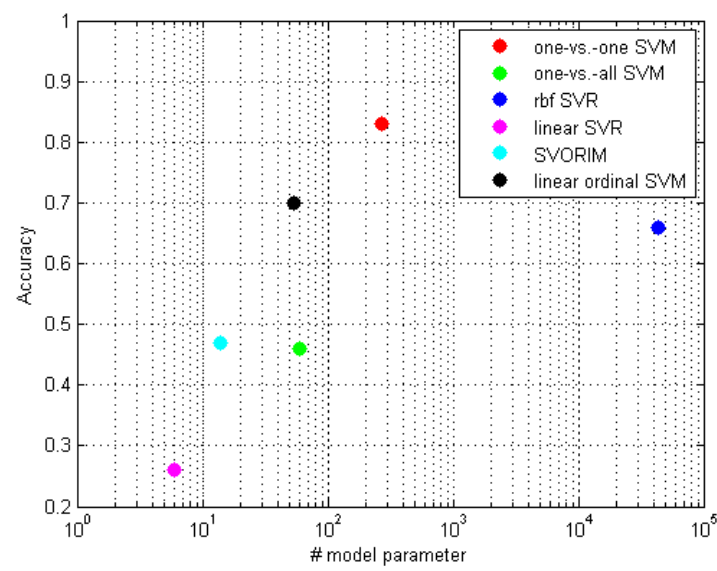

Figure 12: Accuracy related to model size at the example of the barley data set.

\section{CONCLUSION}

We compared the ordinal classification with established algorithms for classification and regression. The ordinal classification turns out to be a high performant method for the classification of ordinal data. The one-vs.-one multiclass SVM is the only method with higher accuracy but this is accompanied by a much higher model complexity resulting in 15 times more evaluation steps. The linear regression methods do not reach a comparable accuracy but they are very compact and fast applied. This example of ordinal data demonstrates that an adaptation of classification algorithms to the specific data characteristics improves the performance drastically. Linear Ordinal SVMs have the potential to be applied in upcoming high-throughput phenotyping facilities which will observe a higher number of plants with a larger spatial and temporal resolution. Especially under limited resources like on unmanned aerial vehicles (UAV) or on mobile devices, it will demonstrate the advantages of including knowledge for compact models.

\section{ACKNOWLEDGEMENTS}

The authors acknowledge the funding of the CROP.SENSe.net project in the context of Ziel 2-Programms NRW 2007-2013 "'Regionale Wettbewerbsfhigkeit und Beschftigung (EFRE)"' by the Ministry for Innovation, Science and Research (MIWF) of the state North Rhine Westphalia (NRW) and European Union Funds for regional development (EFRE) (005-1103-0018) while the preparation of the manuscript. We would like to to thank Christoph Römer, Agim Ballvora and Jens Léon for providing the barley data set.

\section{REFERENCES}

Behmann, J., Steinrücken, J. and Plümer, L., 2014. Detection of early plant stress responses in hyperspectral images. ISPRS Journal of Photogrammetry and Remote Sensing 93, pp. 98-111.
Blackburn, G. A., 2007. Hyperspectral remote sensing of plant pigments. Journal of Experimental Botany 58(4), pp. 855-867.

Chu, W. and Keerthi, S., 2007. Support vector ordinal regression. Neural Computation 19(3), pp. 792-815.

Cortes, C. and Vapnik, V., 1995. Support-vector networks. Machine learning 20(3), pp. 273-297.

Davies, W. J., Zhang, J., Yang, J. and Dodd, I. C., 2011. Novel crop science to improve yield and resource use efficiency in water-limited agriculture. The Journal of Agricultural Science 149(Supplement S1), pp. 123-131.

Dembczyński, K., Kotłowski, W. and Słowiński, R., 2008. Ordinal classification with decision rules. In: Mining Complex Data, Springer, pp. 169-181.

Duan, K. and Keerthi, S., 2005. Which is the best multiclass SVM method? an empirical study. In: Proceedings of the 6th International Workshop on Multiple Classifier Systems, Springer, pp. 278-285.

Exelis Visual Information Solutions, 2012. ENVI 5.0 Help. Exelis Visual Information Solutions, Colorado.

FAO, 2009. Declaration of the world summit on food security. Food and Agriculture Organisation (FAO) of the United Nations, 16-18 November, Rome. [last access: 12.11.2013].

Fürnkranz, J., 2002. Round robin classification. The Journal of Machine Learning Research 2, pp. 721-747.

Gamon, J. and Surfus, J., 1999. Assessing leaf pigment content and activity with a reflectometer. New Phytologist 143(1), pp. 105-117.

Gaspar, T., Franck, T., Bisbis, B., Kevers, C., Jouve, L., Hausman, J. F. and Dommes, J., 2002. Concepts in plant stress physiology. application to plant tissue cultures. Plant Growth Regulation 37(3), pp. 263-285.

Gitelson, A. and Merzlyak, M., 1994. Spectral reflectance changes associated with autumn senescence of Aesculus hippocastanum L. and Acer platanoides L. leaves. Spectral features and relation to chlorophyll estimation. Journal of Plant Physiology 143(3), pp. 286-292.

Guiboileau, A., Sormani, R., Meyer, C. and Masclaux-Daubresse, C., 2010. Senescence and death of plant organs: Nutrient recycling and developmental regulation. Comptes Rendus Biologies 333(4), pp. 382-391.

Kaufman, Y. and Tanré, D., 1996. Strategy for direct and indirect methods for correcting the aerosol effect on remote sensing: from AVHRR to EOS-MODIS. Remote Sensing of Environment 55(1), pp. 65-79.

Kononenko, I., 1994. Estimating attributes: analysis and extensions of relief. Lecture Notes in Computer Science 784, pp. 171182.

Lim, P. O. and Nam, H. G., 2007. Aging and senescence of the leaf organ. Journal of Plant Biology 50(3), pp. 291-300.

Merzlyak, M. N., Gitelson, A. A., Chivkunova, O. B. and Rakitin, V. Y., 1999. Non-destructive optical detection of pigment changes during leaf senescence and fruit ripening. Physiologia plantarum 106(1), pp. 135-141.

Munné-Bosch, S. and Alegre, L., 2004. Die and let live: leaf senescence contributes to plant survival under drought stress. Functional Plant Biology 31(3), pp. 203-216. 
Pennisi, E., 2008. The blue revolution, drop by drop, gene by gene. Science 320(5873), pp. 171-173.

Platt, J., 1999. Advances in kernel methods. Vol. 208, MIT Press, chapter Sequential minimal optimization: A fast algorithm for training support vector machines, pp. 98-112.

Platt, J. C., Cristianini, N. and Shawe-Taylor, J., 1999. Large margin dags for multiclass classification. In: nips, Vol. 12, pp. $547-$ 553.

Rifkin, R. and Klautau, A., 2004. In defense of one-vs-all classification. The Journal of Machine Learning Research 5, pp. 101141.

Smola, A. J. and Schölkopf, B., 2004. A tutorial on support vector regression. Statistics and computing 14(3), pp. 199-222.

Taiz, L. and Zeiger, E., 2010. Plant physiology. 5 edn, Sinauer Associates, Sunderland and Mass.

Tester, M. and Langridge, P., 2010. Breeding technologies to increase crop production in a changing world. Science 327(5967), pp. 818-822.

Tuberosa, R. and Salvi, S., 2006. Genomics-based approaches to improve drought tolerance of crops. Trends in Plant Science 11(8), pp. 405-412.

Vapnik, V., Golowich, S. E. and Smola, A., 1997. Support vector method for function approximation, regression estimation, and signal processing. Advances in neural information processing systems pp. 281-287. 